d'un nettoyage ordinaire de la machine, on recueille un lait contenant presque autant de germes que celui trait à la main.

Les observations ont été renouvelées sur les six vaches de Montafon choisies et accoutumées à la traite mécanique.

Après six jours de traite mécanique normale, on a fait le contrôle pendant deux jours, puis les deux jours suivants, on a opéré la traite à la main.

Le premier jour de la traite de contrôle, on a nettoyé la machine d'après les indications du fabricant, c'est-à-dire qu'on a rincé les tuyaux, a près la traite, à l'eau froide, puis à l'eau bouillante. L'après-midi, la machine a été complètement démontée, et les pièces mises dans une solution alcaline de "radion ». Le deuxième jour, on a modifié le nettoyage en ajoutant $10 \%$ d'eau oxygénée à l'eau froide utilisée pour le rinçage, avant et a près la traite.

Pendant la traite à la main, on n'a pas séparé les premiers jets du lait, mais on a recueilli tout le lait dans le récipient.

Les résultats de ces observations sont consignés dans le tableau ci-joint.

La tranquillité pendant la traite à la machine a eu pour résultat une grande diminution du nombre des germes. Tandis que le lait trait à la main avait dans un gramme une moyenne de 257.444 à 357.888 germes, le lait trait mécaniquement en contenait 93.666 , soit $1 / 3-1 / 4$ des premiers chiffres.

Le résultat a encore été meilleur par l'addition d'cau oxygénée dans les eaux de lavage, le nombre de germes s'étant abaissé à une moyenne de 63.888 pour un gramme de lait.

II résulte que le bon fonctionnement de la trayeuse au point de vue bactériologique est fondé, non seulement sur la tranquillité des vaches pendant la traite, mais aussi sur un bon nettoyage de la machine, meilleur il est, plus grande $\epsilon$ st la diminution du nombre des germes dans le lait, - et qu'avec la trayeuse, on peut obtenir un lait plus pauvre en microorganismes que ne l'est le lait trait à la main.

(Fin.)

\title{
LA MÉTHOdE SYNTHÉTIQUE DANS L'ÉTUDE DU LAIT LE LAIT AU POINT DE VUE COLLOIDAL RECHERCHES SUR LE MÉCANISME DE L'ACTION DE LA PRÉSURE
}

par Ch. PORCHER,

Docteur ès sciences physiques

(Suite)

\section{CHAPITRE VII.}

\section{LE PHÉNOMÈNE DE L'EMPRÉSURAGE}

II_n'est peut-être pas de question qui ait donné lieu à autant de travaux, inspirés d'idées souvent très différentes, parfois inconciliables. 
C'est qu'il s'agissait de donner l'explication d'un phénomène des plus communs, banal pourrait-on dire, d'observation courante, à la portée de tous et qui cependant, pour l'homme de science qui veut le seruter de près, est vraiment d'un intérêt palpitant. C'est une expérience qui étonne toujours que celle qui, consistant à additionner le lait d'une trace infinitésimale de présure, conduit à la transformation plus ou moins rapide du liquide en une masse ferme, au point que l'on peut renverser le tube dans lequel la réaction s'est passée, sans qu'il s'en échappe la moindre goutte. Tout paraît si'simple dans la coagulation du lait de vache par la présure, les contingences qui entourent le phénomène semblent si peu nombreuses, qu'on comprend que ceux qui l'ont étudié, en y apportant plus ou moins d'application, se soient tout naturellement laissé aller chacun à formuler une opinion sur sa nature et son mécanisme. Et c'est ainsi que la littérature qui traite de cette question est devenue considérable ; mais reconnaissons qu'il y entre beaucoup de confusion et de répétitions. Enfin, ajouterons-nous, il en est de la coagulation du lait par la présure, comme de tous les sujets où les conceptions générales peuvent évoluer à l'aise, les explications qu'on en peut donner sont étroitement dépendantes des idées régnantes dans la science. Dans l'historique que nous tenterons de faire un peu plus loin, nous verrons chaque hypothèse emprunter son essentiel aux disciplines du moment de la chimie et de la physique. Et dans le jeu contradictoire des raisonnements qui interviennent pour justifier l'opinion de chacun, il n'est pas surprenant de voir revenir à la surface telle hypothèse qui semblait avoir été antérieurement écartée à tout jamais. Ceci résulte le plus souvent, à n'en point douter, d'une ignorance plus ou moins grande de la bibliographie. Evidemment, on n'est pas sans reculer devant le travail formidable qu'exige le dépouillement des mémoires et des notes qui se rapportent à la question que nous étudions ici ; c'est que s'il en est d'importants, malheureusement il en est bien davantage de plus ou moins insignifiants, desquels ne se dégage aucune idée personnelle digne d'être retenue. Cependant, ce travail doit être fait, car il est vraiment inutile d'enfoncer des portes déjà ouvertes, et e'est ce à quoi trop souvent on s'expose lorsqu'on méconnaît ineonsciemment ou non, les travaux de ses devanciers. Ces réflexions trouveront leur illustration dans les pages qui vont suivre, car nous aurons l'occasion de montrer que si nombre d'auteurs avaient pris soin de se documenter solidement avant leurs recherches et au cours de celles-ci, du temps et de la peine leur eussent été évités, et certaines idées n'eussent point vu le jour.

Nous, n'avons pas la prétention, dans ce travail, de faire une bibliographie complète, - nous avons donné succintement tout à l'heure les raisons pour lesquelles on n'y peut atteindre -, mais du moins nous efforcerons-nous de ne laisser aucun travail important de côté. 
Dans notre premier mémoire (25) sur le lait au point de vue colloïdal, nous aurions dû citer d'autres travaux que malheureusement nous ne connaissions pas à ce moment, parce que nos investigations n'avaient pas porté sur certains périodiques qui jusque-là nous avaient échappé.

Nous souhaitons que beaucoup d'auteurs fassent profit de cet aveu qui s'abrite derrière la meilleure bonne foi, et nous les engageons à faire de plus en plus de la bibliographie. A l'heure actuelle, avee le nombre immense des périodiques paraissant dans le monde entier dans toutes les directions de la science, et plus particulièrement en chimie biologique, il est possible de passer à côté de lectures qui, cependant, devraient être faites. Une organisation du travail scientifique est à réaliser sans tarder.

Les recherches touchant le mécanisme de l'action de la présure sur le lait permettent d'accumuler bea ucoup de faits; parallèlement, elles ne peuvent que favoriser l'éclosion de nombreuses hypothèses. Les faits, - et o'est là une critique qui nous semble permise après nos nombreuses lectures, - sont malheureusement assez souvent mal observés et l'on dirait que les auteurs s'ingénient d'autant plus à multiplier les explications et à les rendre plus embrouillées que s'accentue davantage la carence d'un bon examen des phénomènes.

La complexité de la coagulation du lait par la présure. C'est que, en dépit de sa simplicité apparente, la coagulation du lait par la présure est un phénomène extrêmement complexe. Les contingences qui l'entourent, et que nous disions plus haut pouvoir se ramener à quelques-unes, - ce qui est vrai pour les observateurs superficiels, - sont, au contraire, très nombreuses.

La réaction du milieu, la présence et le taux des sels alcalins et des sels alcalino-terreux, les proportions relatives de ceux-ci et de ceux-là, la solubilité ou l'insolubilité des sels calciques, la quantité de caséine présente, la proportion de l'albumine et de la globuline qui peuvent se trouver en face du caséinate de calcium, la température de la réaction, la présence ou l'absence d'acide carbonique, la quantité de présure mise en œuvre, le chauffage préalable du lait, la dilution, l'absence ou la présence de matière grasse, etc... voilà autant de facteurs qui ont à intervenir. Ils le font chacun dans une mesure différente de celle du voisin, ils peuvent se combiner les uns les autres, tantôt associer leurs actions, tantôt les contrarier. Il importe done pour l'étude de l'influence propre de chacun d'eux d'opérer dans des conditions telles que celles-ci permettent de la dégager clairement et la distraire des actions voisines ou parasites qui peuvent ou l'aider ou l'entraver.

On y peut arriver aisément en ayant recours à la méthode synthétique. Jusqu'ici, l'immense majorité des auteurs, - nous étions sur le point de dire : tous les auteurs, si nous n'avions pensé aux recherches de O. Hammarsten, Laqueur, Courant, Ringer, 一 ont travaillé sur 
le lait lui-même. Nous savons' combien ce liquide est complexe, à la fois solution vraie, solution colloïdale et émulsion, et dans l'enchevêtrement de tous ses composants, il est parfois difficile de s'y retrouver pour attribuer à chacun d'eux la part exacte qui lui revient dans le phénomène de la coagulation par la présure.

L'analyse nous aide à poser des jalons, surtout lorsqu'elle recourt à des moyens non violents qui permettent d'éliminer du lait certains de ses éléments sans porter atteinte aux autres; c'est le cas de la dialyse. Par l'emploi de cette méthode de séparation, on élimine les sels calciques solubles, et on peut alors apprécier leur influence sur la coagulation, en voyant ce que celle-ci devient lorsque ces sels ont disparu. Mais les moyens que l'analyse met à notre disposition sont en somme assez restreints; bien autrement plus féconde est la synthèse. Elle va nous permettre, en partant de la caséine préparée dans un grand état de pureté, de remonter par paliers successifs, graduellement plus chargés de matériaux, à l'édifice entier du lait. Chemin faisant, il devient alors possible de juger de l'influence de chaque principe nouvellement introduit sur la marche et l'aspect de la coagulation.

Avant de faire l'historique critique de la coagulation du lait par ce ferment, nous pensons qu'il est préférable d'examiner très objectivement le phénomène dans tous ses détails.

\section{LA COAGULATION DU LAIT. - COMMENT ELLE SE PRÉSENTE}

Dans toutes les considérations qui précèdent, dès l'instant où nous ne qualifions pas autrement le lait dont nous parlons, il s'agit implicitement du lait de vache.

Il en est de même ici, ce qui se comprend encore mieux, le lait de vache étant la matière première de l'industrie fromagère. Mais les raisonnements qui vont suivre pourraient s'adresser aussi bien au lait de chèvre et au lait de brebis qui servent également à la fabrication de fromages connus et estimés.

$\mathrm{Au}$ surplus, dans cette étude que nous voulons faire porter sur le terrain le plus large possible, nous aurons à examiner comment se comportent d'autres laits, notamment ceux de la femme et des jumentés : jument, ânesse, vis-à-vis de la présure et à expliquer pourquoi ils coagulent si mal ou même pas du tout; les raisons que nous aurons à invoquer permettront même de faire une synthèse de toutes nos acquisitions et de jeter un coup d'œil sur l'influence de facteurs qui jouent plus que les autres dans le phénomène de la coagulation. Revenons done au lait de vache et examinons de la façon la plus attentive ce qui va se passer après l'addition, à une température convenable, - mettons 40 degrés, - d'une certaine quantité de présure. La température à laquelle nos expériences ont été faites, sauf le cas, bien entendu, où 
nous avons voulu apprécier l'influence de températures différentes, a toujours été celle de $40^{\circ}$. C'est, comme nous le verrons, le point optimum; c'est à cette température que pour des quantités constantes de lait et de présure, l'action de celle-ci est la plus rapide et la plus marquée.

II faut travailler sur du lait écrémé. - Nous avons généralement opéré sur du lait écrémé, en dehors bien entendu de l'édifice chimique synthétiquement préparé sur lequel ont porté plus particulière. ment nos recherches de ces dernières années. Lorsqu'on s'adresse au lait entier, l'opacité est plus grande, sa viscosité également, la couleur jaune de la matière grasse dispersée sous forme de globules peut gêner l'appréciation du changement de teinte qu'entraîne la coagulation. Comme la matière grasse n'a pas d'action directe sur l'emprésurage, qu'elle est inutile pour l'étude attentive de ce phénomène, il est recommandé de l'éliminer, de s'adresser toujours au lait écrémé. De petites écrémeuses de laboratoire qui permettent de ne travailler que sur un litre, voire même un demi-litre, donnent toute satisfaction, et s'il est nécessaire, on refait passer deux fois le lait à l'écrémeuse pour le débarrasser presque totalement de ses globules gras.

Le lait qui sort de l'écrémeuse est très mousseux; il faut attendre que la mousse soit affaissée et que le liquide soit redevenu homogène, car, si l'on opérait sur un lait dans lequel se trouvent inclus une infinité de petites bulles pleines d'air, on percevrait mal certains détails. Nous n'insistons pas, pour l'instant, sur la perte d'anhydride earbonique -qu'entraîne le passage à l'écrémeuse et, a fortiori, le double passage, si l'on devait y recourir. Cette perte de $\mathrm{CO}^{2}$ retarde un peu l'emprésurage et nous en donnerons plus tard la raison.

Le lait écrémé de vache se présente avec un aspect blanchâtre sous une épaisseur assez grande, et sous une mince couche avec une translucidité aux reflets bleuâtres que tout le monde connaît.

Ce lait est réparti dans des tubes à essais, à raison de $10 \mathrm{~cm}^{3}$ par tube, et porté wu bain-marie à $40^{\circ}$. Quand l'équilibre de température est atteint, on retire le tube, on l'incline fortement de façon qu'il soit presque horizontal, et on apporte ì l'entrée du tube une goutte de présure, ou ce qui est mieux, une fraction de centimètre eube d'une présure convenablement diluée à l'aide d'une pipette graduée en $1 / 20$ de $\mathrm{em}^{3}$; cette fraction se présente sous la forme d'une goutte ou deux. On relève ensuite le tube doucement, afin de ne pas provoquer à la surface du lait la formation d'une bulle gazeuse toujours difficile à faire disparaître et qui nous gênerait pour l'appréciation exacte du moment où la coagulation débute; puis tout de suite, on reporte le tube au bain-marie. Nous nous excusons d'entrer dans une description aussi minutieuse d'une manipulation vraiment simple, mais on en comprendra les raisons, lorsque nous verrons tout à l'heure qu'il est possible d'opprécier à une ou deux secondes près, avec des emprésurages rapides, le temps qui s'écoule entre l'arrivée de la présure dans le lait et le moment où la coagulation commence. Il importe done de prendre toutes les précautions dont nous avons parlé plus haut 
pour ramener au temps minimum la répartition homogène de la présure dans le lait; en deux secondes pour la quantité de lait mise en œuvre $\left(10 \mathrm{~cm}^{3}\right)$, celle-ci est atteinte. Si l'on est aidé, l'assistant déclanche le chronømètre au moment même où cette répartition est obtenue; c'est le temps 0 à partir duquel nous allons compter les secondes ou les minutes jusqu'au moment où la coagılation va commencer.

LA CoAgulation est un Phénomène continu. - Dès l'instant où la présure est arrivée dans le lait porté à une température convenable, - ici $40^{\circ}$ - elle commence à agir; mals la transformation du lait qui, objectivement, est la plus marquée, en laquelle réside tout le sens du phénomène, est la floculation. Comment se présente-t-elle à nous ?

La floculation. - Si nous sortons le tube de temps en temps avec précaution, - ceci est essentiel - , en le laissant toujours plonger. même partiellement, dans l'eau où il baigne, ce qui ne peut abaisser sa température que d'une fraction de degré, nous pourrons, en suivant l'aspect de la nappe liquide venant couler à l'intérieur du tube, apprécier le moment exact où la floculation commence.

Voici succintement comment les choses se passent. Le lait écrémé qui, en couche mince possède cette translucidité aux reflets bleuâtres bien connue, va blanchir, devenir graduellement plus opaque, les reflets bleuâtres disparaissent, puis il semble que le liquide s'épaississe. C'est que la floculation n'est pas loin; et, en effet, très rapidement, nous voyons qu'à cet épaississement de la liqueur, la rendant moins mobile, fait suite l'apparition de petits flocons, d'abord séparés les uns des autres, et qui vont se souder pour former le bloc ferme sur lequel ultérieurement la synérèse va s'exercer.

Il est donc trois phénomènes qui se suivent avec plus ou moins de rapidité : $1^{\circ}$ Le lait blanchit davantage, il s'opacifie, puis 20 il s'épaissit, et enfin $3^{0}$ apparaissentles petits flocons qui marquent le début dela coagulation. Nous disons que le lait blanchit; la chose pourra paraître singulière, puisque le lait écrémé est déjà blanc et cependant, ce point n'est pas contestable. Ce changement de teinte serait difficilement perçu, autant dire, pas du tout, si l'on devait se contenter de regarder la masse du liquide par réflexion; mais dès l'instant où l'on fait porter son attention sur une lame mince du liquide, celle qui s'écoule à l'intérieur du tube lorsqu'on l'incline avec précaution, on juge très facilement de la suite des phénomènes que nous venons de décrire. La tendance à l'opacité s'accentue graduellement; c'est elle qui demande le plus de temps, puis la lame liquide s'épaissit; e'est assez rapidement que cette modification se fait; elle est suivie très vite également de la floculationL'épaississement doit être surveillé de près, puisqu'il est le prélude de la floculation qui ne saurait tarder, surtout dans les coagulations rapides. Dans ces conditions, en effet, le passage de l'épaississement de la couche 
opaque à la floculation, laquelle se traduit très exactement par une dispersion de petits flocons de forme irrégulière au sein d'un sérum limpide, est extrêmement rapide et on peut la saisir quelquefois à une seconde près, deux secondes tout au plus.

Evidemment, avec les emprésurages lents, les phénomènes dont nous venons de parler s'étalent sur une période plus longue et on saisit moins facilement leur succession, le passage de l'un à l'autre. La floculation tarde à se déclancher et dans ce cas, si une petite erreur de mesure de temps entre en jeu au point de vue absolu, elle est, au point de vue relatif, pour ainsi dire négligeable. Mais pour les coagulations rapides demandant quelques dizaines de secondes, une ou deux minutes au plus, il importe de suivre très attentivement les phases successives de l'emprésurage, telles que nous venons de les décrire.

La prise en masse. - Si l'on ne prête pas à la coagulation du lait toute l'attention voulue, si l'on n'en suit pas les phases, seconde par seconde, on ne verra seulement que ce qu'on peut appeler la prise en masse, sans avoir pu se rendre compte que celle-ci, après tout, ne résulte que de la soudure rapide de flocons antérieurement formés.

Pour Spring qui a beaucoup étudié les phénomènes de la coagulation des colloïdes, la prise en masse serait le fait général, et les flocons résulteraient pour lui d'une brisure ultérieure par agitation.

Cette hypothèse qui est valable dans certains cas ne saurait être admise pour le lait. Nous verrons en effet, toutes les fois que l'emprésurage est gêné, - nous aurons à montrer pourquoi, - que les flocons se soudent plus ou moins facilement; quelquefois même, ils ne se soudent point. Il se forme des petits agglomérats, mais la prise en masse n'est pas constatée. Bref, dans tous les cas, que l'emprésurage soit normal ou anormal, la forme floculante est toujours la forme initiale, mais la coagulation ne va pas toujours jusqu'à la forme gélifiante,

L'importance de la masse de lait écrémé soumise à l'emprésurage n'exerce aucune influence sur la marche de la coagulation, et-la suite des. temps que nous venons d'examiner, un à un, est la même, qu'il s'agisse de surveiller l'emprésurage de 2.000 litres de lait dans une cuve ou celui de $10 \mathrm{~cm}^{3}$ dans un tube à essais.

Ce qui est aussi caractéristique, ou presque, que la rapidité de la floculation, ou disons mieux, la brusquerie avec laquelle les flocons apparaissent dans les cas d'emprésurage rapide, c'est leur soudure. également rapide. Ces flocons très petits avec l'emprésurage lent, pares. seux, comme on dit encore, sont plus gros avec les emprésurages vigoureux; ils sont de formes irrégulières, semblent plutôt être constitués de lamelles, et leur plasticité est telle que, dès leur apparition à l'état dispersé au sein du sérum limpide, ils ne tardent pas à se souder les uns aux autres pour former un bloc compact qui occupe totalement le volume initial du liquide. C'est le caillé ou coagulum; il apparaît très 
homogène lorsqu'il vient de se former, mais, plus ou moins rapidement, il va se contracter, abandonner la paroi du tube à essais, dans toute sa hauteur et sur tout le pourtour, et laisser exsuder du sérum limpide.

La Synérèse. - La rapidité avec laquelle s'effectue cette contraction appelée synérèse dans le langage colloïdal, dépend de plusieurs facteurs que nous examinerons plus loin, mais nous citerons déjà l'acidité parmi ceux qui ont le plus d'influence.

LA STRUCTURE DU " CAILLÉ ". - On peut comparer le caillé à une éponge très fine dont les travées serrées les unes contre les autres circonscrivent des logettes où le sérum se rassemble; ces travées, du fait de leur plasticité, se rétractent, ce qui détermine un excès de pression à l'intérieur des petites logettes et oblige le liquide qui s'y trouvait rassemblé, à les quitter.

La comparaison du caillé avec une éponge n'est pas une image. S'il est difficile, dit J. Duclaux, de donner la preuve de la structure spongieuse de la plupart des coagulats de colloïdes, nous dirons que, pour le lait, il s'agit d'une réalité, car nous verrons, lorsque nous ferons porter l'action de la présure sur des associations de caséinate de calcium avec d'autres sels, calciques, que le coagulum qui se forme n'est pas toujours massif comme dans le cas du lait écrémé, mais réellement spongieux. A l'œil nu, on voit des trabécules orientées en tous sens, de l'entrecroisement desquelles résultent des petites cavités bien formées qui logent le sérum. La synérèse est dans ce cas extrêmement rapide, et lorsqu'on détache le caillé du tube, on y trouve une texture filamenteuse qui résulte en quelque sorte de la soudure des travées du coagulum aplaties les unes contre les autres, après l'exsudation du sérum.

COMMENT DEFINIR LE TEMPS DE LA COAGULATION ? - Après l'examen que nous avons fait de tout ce qui objectivement peut être saisi dans la coagulation du lait, nous allons pouvoir définir ce qu'il faut entendre parle temps de coagulation. C'est exactement le temps qui s'écoule entre le moment où le chronomètre a été déclanché lors de l'apport de la présure dans le tube et après sa répartition homogène dans le liquide, et celui où la floculation apparaît. Nous avons vu, - et l'expérience est à la portée de tous ceux qui veulent la faire, - que l'on peut estimer avec une grande précision l'instant où la floculation se déclanche; c'est bien à une ou deux secondes près qu'on la saisit. Pour un phénomène biologique, on ne saurait vraiment mieux obtenir. Si nous insistons comme nous venons de le faire, c'est parce qu'il est essentiel de déterminer avec une grande exactitude la durée de la coagulation, du moment que nous avons à la faire entrer dans certaines équations à côté de la masse du lait et de la quantité de présure, pour l'étude de la loi d'action de celle-ci. 


\section{La critique du mesurage de la présure par gouttes. - Au} début de nos recherches, nous employions la présure par gouttes, mais nous nous aperçûmes très vite, alors même que nous nous servions d'un comptegouttes étalon, tel le compte-gouttes Ductaux, que c'était là un mode défectueux de mesure parce que le poids des gouttes n'est pas toujours le même selon l'espèce de présure que nous utilisons. En effet, selon que la solution de présure contient des sels ou en est, au contraire, débarrassée, est plus ou moins diluée, la tension superficielle varie, et conséquemment, avec elle, le poids des gouttes. Enfin, on voudra reconnaître avec nous, que même en se servant d'un compte-gouttes étalon, il peut arriver que l'on ait affaire à des gouttes de poids différents, parce qu'on les fait écouler plus ou moins vite, avec plus ou moins de brusquerie, et comme il s'agit d'une solution d'une diastase extrêmement active, il suffit, on le conçoit, d'une différence extrêmement minime pour influencer nettement le temps de la coagulation. Aussi, avons-nous substitué au mesurage par gouttes, le mesurage en volume avec des pipettes bien calibrées de 1 centimètre cube divisées en vingtièmes. L'erreur sur le mesurage est moindre, et nous prenons soin pour la diminuer encore de nous adresser à des présures fortement diluées.

\section{LES PRÉSURES EMPLOYÉES}

La solution du ferment est préparée, au laboratoire, par macération de caillettes de veau dans de l'eau chlorhydrique à $40 / 00$ ou bien nous utilisons des présures commerciales, soit liquides, soit en poudre.

MACERATION CHLORHYDRIQUE DES CAILLETTES. - Il est difficile, pour ne pas dire impossible, d'être assuré d'avoir des portions de caillettes qui, à poids égal, aient bien la même richesse en ferment. Pour nos recherches, nous nous sommes adressé à des caillettes fort belles, d'épaisseur uniforme, débarrassées des parties conjonctives et graisseuses qui encombrent souvent les caillettes de qualité inférieure ; bref, nous nous sommes entouré du maximum de précautions pour être certain d'avoir des languettes homogènes et semblables les unes aux autres. Les macérations de caillettes dans l'eau chlorhydrique à 4 p. 1000 sont faites à des températures ordinaires ou basses, parce que si l'on porte à l'étuve, très rapidement la caillette se digère et on ne trouve plus qu'un liquide trouble dans lequel nagent des filaments du tissu élastique qui n'a pas été attaqué. Avec l'acidité du milieu et la présure, nous avons, en effet, tout ce qu'il faut pour digérer très vite le tissu facilement digérable de la caillette, c'est-à-dire la muqueuse. N'est-ce pas de nouveau la preuve qu'il est difficile de faire une distinction nette entre la présure et la pepsine et que seule la réaction du milieu conditionne l'action de l'une ou l'autre. On dit que c'est la présure qui agit lorsque la réaction du milieu est voisine de la neutralité potentiométriquement déterminée ; on dira par contre que c'est la pepsine, quand il 
s'agit d'une acidité plus marquée et que le coagulum est l'objet d'une dialyse rapide. Avec de l'eau chlorhydrique à 4 p. 1000, nous réalisons là le taux d'acidité du suc gastrique lui-même; ne soyons donc pas surpris si la macération de caillettes dans une pareille eau acide et portée à $40^{\circ}$ soit rapidement digérée.

Lorsqu'on fait macérer une caillette dans de l'eau chlorhydrique à basse température, la languette mince se gonfle; on voit très bien sur la tranche, la musculeuse se séparer de la muqueuse plus épaissie et plus translucide, mais le liquide dans lequel baignent ces languettes de caillettes reste toujours limpide. Au bout de 24 heures, et même bien avant, la macération est extrêmement active vis-à-vis du lait et il suffit d'une quantité infime pour coaguler $10 \mathrm{~cm}^{3}$ de lait à $40^{\circ}$ en moins de 10 à 15 secondes. Cette présure que l'on apporte est accompagnée, évidemment, d'une acidité ionique assez forte, mais qui se trouve très diluée dans le lait; néanmoins, en dépit de cette dilution, elle joue un rôle important, comme nous verrons plus loin.

Macération de caillettes de veaux à des titres divers. Quatre macérations de languettes de caillettes sont faites dans de l'eau chlor. hydrique à 4 pour 1000 :

a) 1 gr. dans $100 \mathrm{~cm}^{3}$ d'eau chlorhydrique

b) $2 \mathrm{grs}$

c) $5 \mathrm{grs}$

d) $10 \mathrm{grs}$

On porte à la glacière et on laisse 24 heures. A $20 \mathrm{~cm}^{3}$ de lait écrémé chauffé au préalable à $40^{\circ}$ on ajoute un $1 / 2 \mathrm{~cm}^{3}$ de la macération filtrée et on reporte au B.M.

Voici les temps de prise :

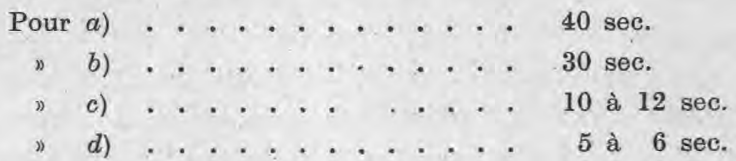

Comme les deux dernières dilutions sont trop corcentrées et que l'erreur sur le temps de prise, n'atteindrait-elle qu'une seconde ou deux, est encore plus grande en valeur relative qu'elle ne l'est en valeur absolue, nous diluons les macérations $b$, $c$ et $d$, pour les ramener au même taux initial de macération de languettes que la macération $a$. En conséquence, la macération $b$ a été additionnée de son volume d'eau, la macération $c$, de 4 volumes, la macération $d$, de 9 volumes.

Voici ce que deviennent ces temps de prise après ces dilutions :

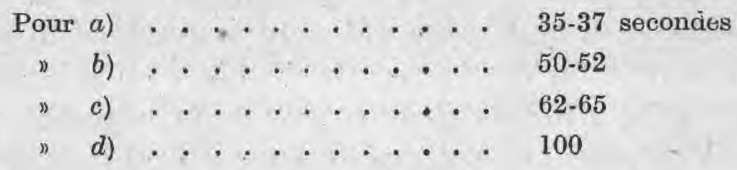


Si on divise ces temps par le taux de la dilution, on a :
a) $35-37 \mathrm{sec}$.
b) $25-26$
c) $12-13$
d) 10

Il résulte de cette expérience fort simple que la force des dilutions, en d'autres termes, la richesse des macérations initiales en présure, n'est nullement proportionnelle au poids de la caillette employée.

La macération des caillettes de veaux, dans l'eau chlorhydrique à 4 pour 1000, n'extrait pas, si prolongée qu'elle soit, tout le ferment que la muqueuse renferme.

La «force » de la présure en fonction de la durée de macération. - Nous avons pris 20 grammes de caillettes que nous avons fait macérer après les avoir découpées en languettes dans $500 \mathrm{~cm}^{3}$ d'eau chlorhydrique à 4 pour 1000.

Le lendemain à la même heure, done après 24 heures de macération, nous déterminons le temps de prise, en secondes, de $20 \mathrm{~cm}^{3}$ de lait écrémé, préparé avec la poudre Krause, additionné de $1 / 2 \mathrm{~cm}^{3}$ de la macération filtrée et diluée, de façon que la première prise se fasse entre 4 ou 5 minutes.

Les $2^{\mathrm{e}}, 3^{\mathrm{e}}, 4^{\mathrm{e}}, 5^{\mathrm{e}}$ jours, à la même heure, nous faisons des prises de la même macération que nous faisons agir sur le lait écrémé préparé de manière identique.

Voici les résultats obtenus donnés dans le tableau XXXIV d'abord, traduits par le graphique II ensuite.

TABLEAU XXXIV.

TEMPS DE PRISE DU LAIT PAR DES MAGÉRATIONS DE GAILLETTES D'AGES DIFFÉRENTS

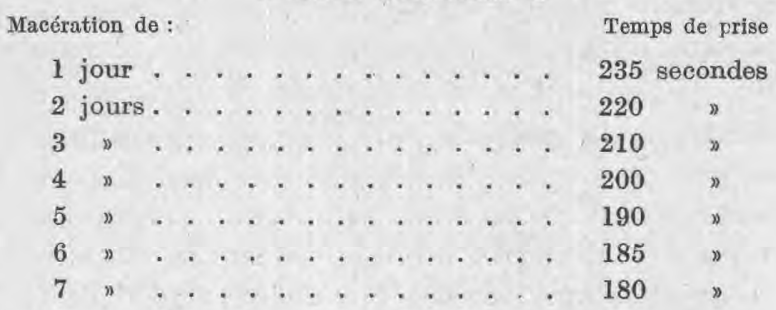

On se rend compte que la durée de la macération n'a que peu d'influence sur la force de la présure. Si la macération, le septième jour, fait faire prise au lait un peu plus vite, sa force n'est cependant pas augmentée comme on aurait pu le penser a priori.

LA diALYSE des solutions de pRÉsure. - L'emploi d'une macération chlorhydrique de présure ne peut que conduire à des coagulations rapides. Pour éliminer l'acide chlorhydrique présent, il est indiqué de dialyser cette présure contre un courant d'eau de la conduite d'abord et, pour terminer, car l'eau de la conduite est toujours calcaire, contre de l'eau distillée. 
GRAPHIQUE II.

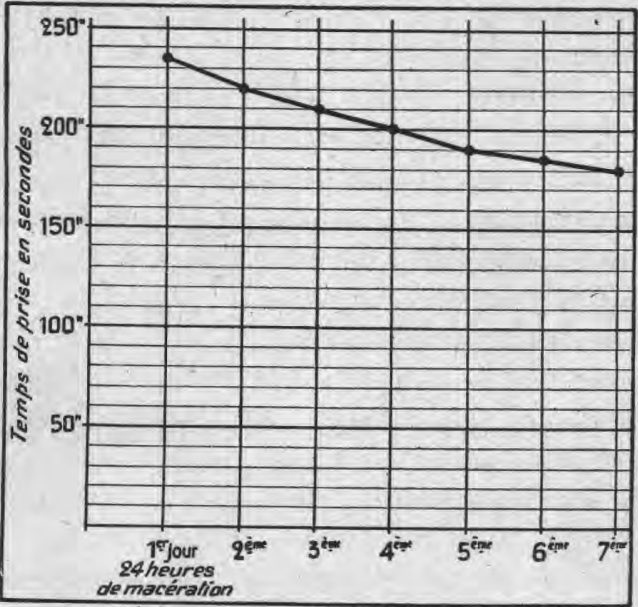

Lorsqu'on s'adresse aux présures commerciales, liquides ou en poudre, qui sont très chargées de chlorure de sodium, la quantité de ce sel est souvent telle que, dans des recherches ou l'on doit se garder de l'apport d'un sel alcalin, il est de toute obligation de ne pas recourir à ces présures, à moins qu'elles ne soient dialysées pendant un temps suffisant, d'abord contre l'eau de la conduite, ensuite contre de l'eau distillée, pour en éliminer tout le chlorure de sodium, et n'y laisser que des traces de matières salines, sans doute insolubles, lesquelles vraisemblablement ne jouent plus qu'un rôle très restreint, si ce n'est même aucun rôle, puisque le peu de présure qu'on emploie se trouve encore fort dilué dans la quantité de lait qui reçoit le ferment.

La sensibilité de la présure aux rayons chimiques. - Une observation importante doit être faite, surtout lorsqu'il s'agit d'apprécier la force d'une présure. Lorsqu'une solution de présure est si active qu'il a été indiqué de la diluer au préalable, il faut se garder de la laisser au soleil, parce qu'assez rapidement elle s'altère et perd de sa force, le ferment étant graduellement attaqué sous l'action des rayons chimiques. Toute solution de présure dont on se sert au laboratoire dans la journée pour des recherches délicates doit être conservée à la glacière, à l'obscurité, bien entendu, si on veut l'utiliser le lendemain et les quelques jours qui suivent.

LE LAIT D'UNE MÊme VACHE VIS-A-VIS dE LA PRÉSuRE. - En dépit de ces précautions, on ne saurait toutefois comparer, sur plusieurs jours, les temps de la coagulation, parce que la présure a pu s'affaiblir un peu, bien qu'on l'ait conservée à l'obscurité et au frais.

Une difficulté nouvelle surgit à propos du lait cette fois. Lorsqu'on 
fait un édifice synthétique à base de caséine du genre de ceux dont nous parlerons ultérieurement, nous sommes absolument maître des conditions de son obtention, et l'on peut être certain de travailler sur un ensemble toujours le même. Mais pour le lait, il en va tout différemment, et serait-ce même le lait de la même vache qui serve à toute une suite d'expériences sur plusieurs jours consécutifs, il n'est pas vrai de dire que c'est toujours le même lait, en admettant encore une fois qu'on l'ait écrémé et que l'on se contente d'opérer sur la partie dégraissée. En effet, si c'est au lait entier que l'on s'adresse, ce n'est pas le même lait, du fait des variations de sa matière grasse ; mais laissons la matière grasse de côté et ne considérons toujours que la partie non grasse. Le lait écrémé d'un animal en bonne santé, soumis à un régime régulier se présente dans ses grandes lignes comme un ensemble à peu près fixe de composition, avec, cependant, des petites variations qui, si elles ne touchent pas à ce que nous avons appelé naguère la quasi-fixité de l'extrait oégraissé $(\mathbf{2 8})$, sont toutefois très bien perçues par la présure. Des mêmes quantités de lait écrémé des traites successives d'une même vache ne coaguleront pas dans le même temps avec la même présure. En conséquence, lorsqu'on fait des expériences comparatives, d'ordre purement expérimental, sur un lait donné dont on modifiera, ou la réaction ou l'apport calcique, les expériences devront être faites dans la même journée.

L'emploi du lait desséché dans l'étude de la présure. - Si le lait d'un même animal, dont l'alimentation et l'entretien ne subissent pas de modifications sensibles ne semble pas d'un jour à l'autre réagir absolument de la même manière vis-à-vis de la même quantité de la même présure, on ne peut s'en servir comme étalon pour apprécier des présures différentes au cours d'une expérience de plusieurs jours. Il nous parait cependant indispensable d'avoir en mains un lait qui puisse être semblable à lui-même sur une longue période de temps. Nous pensons l'avoir trouvé dans le lait écrémé reconstitué en partant de la poudre de lait écrémé préparée par le procédé Krause. Cette poudre, en effet, est soluble dans l'eau et les conditions de son obtention sont telles (221) que le trouble apporté à l'harmonie de la composition du lait originel, à l'équilibre de son édifice salin, est minimum pour ne pas dire négligeable.

Voici comment nous utilisons cette poudre :

10 grammes sont dissous dans une quantité d'eau suffisante pour faire $100 \mathrm{~cm}^{3}$; nous réalisons ainsi un lait écrémé tout à fait comparable à celui que nous donnerait le passage à la centrifuge d'un lait frais normal, car il faut tenir compte ici du taux d'humidité que renferment toujours les poudres ; l'extrait sec du lait reconstitué approche 94 à 95 grammes par litre

Sans addition aucune de chlorure de calcium, ce lait reconstitué coagule assez bien par la présure, mais si l'on veut être assuré d'obtenir une coagulation toujours

(221) Ch. Porcher. - Le Laít desséché, $2^{\circ}$ édition, 1926, p, 11. Le Laít, Ed. Lyon. 
ferme, meilleure en quelque sorte, nous recommandons de l'additionner d'une quantité de chlorure de calcium égale à 1 gramme par litre ; quelquefois, on peut se contenter de 0 gr. 50.

Avec la poudre de lait, on peut ainsi être assuré d'obtenir un lait liquide, reconstitué qui soit semblable à lui-même sur un grand nombre de jours, un temps suffisant du moins pour rendre tout à fait comparables les résultats des recherches entreprises.

La poudre Krause est une poudre obtenue par "brouillard"; les poudres obtenues sur cylindres chauffés et qui, de ce fait, ont été soumises à une température plus élevée ne permettant pas une redissolution aussi parfaite, nous ne les recommanderons pas dans les expériences de la nature de celles qui sont relatées ici.

\section{LA FORCE DE LA PRÉSURE}

$\mathrm{Au}$ cours des recherches faites sur la présure, on se rend compte qu'avec des volumes égaux de solutions de celle-ci et des quantités égales de lait, on n'obtient pas des coagulations dans les mêmes temps. C'est done reconnaître que les préparations de présure ne renferment pas les mêmes quantités de ferment.

Ne sachant pas encore préparer la présure à l'état pur, il est donc impossible, - nous en dirions autant de toutes les autres diastases d'avoir des solutions dont le titre en ferment puisse être pondéralement déterminé. Nous ne pouvons apprécier le taux de la présure que par la quantité de lait qui est coagulée ; mais comme la gélification du lait est également fonction de la température, - nous ne parlons pas pour l'instant de la réaction du milieu, - il faut évidemment que ce facteur intervienne; nous aurons donc à en tenir compte dans la définition de ce qu'on est convenu d'appeler la force de la présure.

Définition de la ( force "d'une présure. - Sous le nom de force d'une présure, on désigne le nombre en volume de parties de lait qui sont coagulées par une partie de présure en 40 minutes, la température étant maintenue à $35^{\circ}$.

C'est cette définition très empirique que l'on trouve dans tous les ouvrages ; elle est à la base de l'appréciation de la valeur des préparations commerciales.

L'intervention du facteur temps a été mise en lumière, il y a fort Iongtemps, par Storch et SEgelCKe (222). Ce sont les premiers auteurs qui aient cherché à enfermer la marche de l'emprésurage dans une formule traduisant le texte d'une loi. Pour eux, le temps de coagulation est inversement proportionnel à la quantité de ferment employée; autrement dit: le produit de la quantité de ferment par le temps de la coagulation est un nombre constant.

(222) Storoh et Segelcke. - Versuche über das Gerinnen der Milch. Milchzeitung. $1874,3,997$. 
Dans cette formule, le temps est calculé en secondes, la concentration de la présure en quantité de présure pour cent.

II s'agit là d'une loi simple, mais les recherches qui ont été faites ultérieurement ont montré qu'elle ne s'observait pas d'une façon régulière et qu'elle subissait de nombreux écarts.

SOXHLET (223) confirme ee qu'il appelle l'exactitude de la loi de STORCH, lorsque les temps de coagulation vont de 2 à 40 minutes. C'est à partir des recherches de cet auteur que la détermination de la force de la présure dans les préparations commerciales a revêtu l'allure qu'on lui connaît.

Cependant, comme le font remarquer W. GrTmmer et M. KrUGER (224) si l'on dépouille avec soin le document de SoxHLET, - car dans le travail de Storch et SkgkLcke, il ne s'agissait que de trois expériences, - on constate que la loi de STORCH et de SEGELCKE n'est pas observée, puisque lorsque la quantité de présure augmente, les produits augmentent légèrement. Dans le travail de Lörcher (225), il en est de même bien que cet auteur conclut que la loi de STorch lui paraît également confirmée.

Les travaux s'accumulent amenant leur cortège inévitable de manque de concordance dans les résultats des auteurs qui se sont attaqués à la vérification de "la loi de Storch. Pour les uns : Lörcher (225, Peters (226), Kônig (227), Alteiman et Schmidt (228) lorsque la concentration de la présure diminue, les produits diminuent; pour les autres : MAYER (229), HiLmmanN (230), lorsque la concentration de la présure diminue, les produits augmentent; enfin, et c'est la conclusion de Th. MaDsen : lorsque la concentration de la présure diminue, les produits commencent par diminuer, puis ils augmentent.

Plusieurs auteurs, GRIMmer et KRÜGER, LENK (231) se sont employés à expliquer ces contradictions, et ils insistent, avec raison

(223) F. SoxнLет. - Die Darstellung baltbarer Labflüssigkeiten. Milchzeitung, 1877, 6, 495 et 513 .

(224) W. Grimmer et M. Krüger. - Beiträge zur Kenntnis der Labwirkung-. Milch. Forsch. 1925, 2, 457 .

(225) Lörcher. - Ueber Labwirkung. Arch. f. Physiol. 1897-1898, 69, 141.

(226) Peters. - Untersuchungen über das Lab und Labähnliche Fermente, Preischrift, Rostock, 1894.

(227) A. KöNIG. - Untersuchungen über den Einfluss von Temperaturen auf Fermente besonders von Lab und Pepsin. Biochem. Zeitschr. 1920, 110, 266.

(228) A. Alifman et H. Schmidt. - Ueber die Festigkeit des durch Lab erzeugtern Milchkoagulums. Landwirtschaftl. Jahrbuch d. Schweiz, 1916, 30, 355, et Milchwirts. Zentralblatt, 1916, 45, 273, 292 et 305.

(229) MAYER. - Bestimmungen der Wirksamkeit des Labfermentes unter versehiedenen âusseren Umstânden. Milchzeitung, 1881, 12,17 et 33.

(216) Hinsmain. - Arbeiten des landwirtschaftlichen Institutes der Universität, Leipzig, 1897, 1, 113.

(231) E. LENK. - Rovision des Labgesetzes. Biochem. Zeitschr. 1926, 178, 105. 
pensons-nous, sur la difficulté d'apprécier exactement le temps exigé pour la coagulation du lait. Parfois, on se contente de le mesurer en minutes et en demi-minutes et on ne pousse pas jusqu'aux secondes. Personnellement, nous estimons que pour les coagulations rapides, et pour celles qui sont extrêmement lentes, si la loi de STORch ne se vérifie pas, c'est peut-être, pour une grande part, le fait d'une erreur dans le temps d'appréciation exact du moment de la coagulation, Pour les coagulations extrêmement rapides demandant moins d'une demi-minute, quelques secondes même, une erreur faible dans la mesure du temps entraîne une erreur importante dans le produit.

Dans les emprésurages qui tardent, emprésurages paresseux, on peut avoir des erreurs grandes dans l'appréciation des temps. Si l'on estime que la coagulation doive aller jusqu'à la prise du lait en bloc, on se trompe certainement, parce que, bien avant que la gélification totale ait été obtenue, il y a floculation, le bloc du gel ne résultant, comme nous l'avons vu, que d'une soudure ultérieure de flocons primitivement dispersés ; cette soudure qui est fort rapide dans les emprésurages eux-mêmes rapides, demande un certain temps dans les emprésurages lents avant d'arriver au bloc compact, Dans la vérification d'une loi biologique, comme celle de SтоRсн, il importe donc de ne pas faire d'erreur appréciable dans la détermination du moment où la floculation apparaît, car c'est elle qui est le phénomène essentiel, et toute erreur absolue dans la mesure du temps se traduit dans le prcduit par une erreur relative qui peut être considérable.

LENK prend soin de remarquer que si la coagulation demande plus de 20 minutes, on commettra toujours une grosse erreur dans l'appréciation des résultats, par suite, dans la mesure des produits du temps par la quantité de présure employée. Il reconnaît également que dans la zone moyenne, c'est-à-dire pour des temps de coagulation moyens, les produits sont sensiblement voisins les uns des autres et paraissent justifier l'exactitude de la loi de STORCH.

Grimmer et KRÜGer semblent dénier toute valeur à celle-ci. Pour eux, les produits augmentent, même lorsque la concentration en présure augmente, et les produits sont une fonction exponentielle de la quantité du ferment; ils suivent une courbe logarithmique qui se rapproche asymptotiquement d'une valeur maximum.

Les résultats auxquels GRTMMER et KRÜGER sont arrivés se traduisent de la façon suivante: lorsque les temps de coagulation sont grands, les produits sont assez voisins, mais dès que les temps de coagulation commencent à être plus petits, les produits augmentent incontestablement; il arrive même que le dernier produit peut être le double du premier, ce que l'on traduira en disant que la vitesse de coagulation est deux fois moindre que lorsqu'on emploie de faibles quantités de présure ou, encore, que la force de la présure semble dimi- 
nuer à mesure que les quantités de ferment devant agir sont plus grandes.

On sait que si l'on veut traduire dans une courbe l'action de la plupart des ferments, on constate que cette action est une fonction logarithmique de la quantité de ceux-ci. Si l'on cherche à retrouver la même expression graphique pour la présure, on n'arrive à aucun résultat; par contre, ce sont les produits de la quantité de présure par

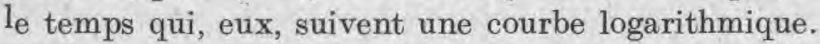

On traduira toutes ces observations en disant que la force de la présure diminue alors que la quantité de présure augmente. Il en résulte donc que si la quantité de présure augmente, la vitesse avec laquelle agit le ferment diminue. Cette diminution, pour des quantités très élevées de ferment, s'approche d'une valeur minimum.

Dans les recherches de GrTMmer et de KrüGER, ainsi que dans celles de beaucoup d'auteurs, et les nôtres propres, il y a souvent une grande dilution du lait. A un volume déterminé de lait, on apporte un volume d'une solution de présure en représentant souvent le quart ou la moitié. Il en résulte done une dilution de la caséine et, surtout, si on ne prend pas de précautions, un refroidissement de la masse. II est done recommandé de mélanger le lait et la solution de présure, chauffés préalablement l'un et l'autre à la température de l'emprésurage, soit $35^{\circ}$, pour les expériences qui portent sur la détermination de la force de la présure.

Cette forte dilution du lait par la solution de la présure est peut-être un inconvénient. C'est pourquoi dans ses recherches, LeNK détermine la force de la présure sur la base de la loi de STORCH selon un procédé dû à Dewarda :

On prend $200 \mathrm{~cm}^{3}$ de lait - quantité de lait plus importante que celle employée par Grtmmer et KrüGer - qu'on porte à $35^{\circ}$ et on y ajoute $2 \mathrm{em}^{2}$ d'une solution de présure à $1 / 20$.

On calcule la force de la présure d'après les considérations suivantes :

On dit qu'une préparation commerciale de présure liquide a une force de 10.000 , force la plus courante, lorsque $1 \mathrm{~cm}^{3}$ de cette présure coagule en 40 minutes à $35^{\circ}, 10.000 \mathrm{~cm}^{3}$ de lait.

' Si cette solution est diluée au 1/20 et qu'on en prenne $2 \mathrm{~cm}^{3}$ pour $200 \mathrm{~cm}^{3}$ de lait, la force de la présure sera égale à : $\frac{40 \times 2000}{t}$ ( $\mathrm{t}$ en minutes) puisque 2000 est le rapport de la quantité de lait à la quantité de présure utilisée : $\frac{200}{2}$ et 40 , le temps en minutes de la coagulation étalon.

$2 \overline{0}$

LENK utilise également des quantités faibles de lait : $10 \mathrm{~cm}^{3}$. Peutêtre qu'avec cette quantité, le temps de prise est toujours légèrement inférieur à celui qu'on pourrait mesurer en utilisant $200 \mathrm{~cm}^{3}$, ce qui selon nous, est dû à la facilité plus grande à déterminer à la seconde 
près parfois pour des présures actives le moment où la coagulation commence.

LENK remplace l'expression force de la présure par celle d'unités de présure. Il en calcule le nombre de la façon suivante :

Si pour $10 \mathrm{~cm}^{3}$ de lait, on emploie $1 \mathrm{~cm}^{3}$ d'une solution de présure au $1 / 100$, le rapport de la quantité de lait à la quantité de présure est de 1000/1 et la valeur U. P. (unités de présure) est obtenue de la façon suivante : si le lait coagule en 4 minutes ou 240 secondes, au lieu de 40 minutes, l'extrait de présure est de $1000 \times 10=10.000$ U. P. En d'autres termes, en divisant 24 par le nombre de secondes et en multipliant le quotient par 100.000 , on obtient la force en U. P. :

$$
\text { U. } \mathrm{P} .=\frac{40 \times 1.000}{\mathrm{~m} \text { (minutes) }}=\frac{24 \times 100.000}{\mathrm{~s} \text { (secondes) }}
$$

LENK estime que pour des coagulations allant de 6 à 14 minutes, la loi de STORCH est à peu près exacte. GRIMMER et KRÜGER allaient jusqu'à observer des temps de coagulation qui étaient de 30 minutes; c'est ce que leur reproche LENK en faisant remarquer qu'il s'agit là de temps vraiment longs et pouvant conduire à des conclusions généralement inexactes. Dans les zones moyennes de temps de coagulation et de concentration de présure, la loi de STORCH conserve toujours sa valeur pour l'appréciation des préparations commerciales.

L'INFLUENCE dE LA DiLUtion de LA PRÉsure. - La présure qui a été utilisée est la présure liquide.

On prend $10 \mathrm{~cm}^{3}$ de lait et on y ajoute une goutte de présure (a).

Voici les résultats obtenus dans une première série d'expériences :

\section{TABLEAU XXXV.}

INFLUENGE DE LA DILUTION DE LA PRÉSURE SUR SA “ FORCE ".

Dilutions
$\begin{array}{ll}\text { Non diluée } \\ \text { Diluée à } & 1 / 2 \\ \text { " } & 1 / 3 \\ " & 1 / 4 \\ " & 1 / 5 \\ \text { " } & 1 / 6 \\ \text { " } & 1 / 10 \\ \text { " } & 1 / 15\end{array}$

Temps de prise

104 sec.

236

369

431

574

627

1015

1850
Produit cu temps de prise par le taux de la dilution

104

118

123

108

115

106

101

123

On ne saurait vraiment dire, en s'appuyant sur les chiffres de la

(a) Présure Fubre. Que la Maison Fabre veuille bien agréer ici mes vifs remerciements. (Ch. P.). 
dernière colonne, que le produit du taux de la dilution par le temps de prise est un nombre constant. Les grands écarts que nous constatons doivent tenir à ce que nous avions d'abord mesuré la présure employée par gouttes; or nous avons fait la critique de cette façon de faire.

Dans une deuxième série d'essais, on opérant avec la même présure non diluée, nous avons eu des temps de prise successivement de : 127 secondes, 152, 140, ce dernier nombre étant la moyenne des deux premiers.

Nous avons fait une troisième série d'essais sur des dilutions successives de la même présure, mesurées cette fois avec une pipette graduèe au $1 / 20$ de $\mathrm{cm}^{3}$, et non par gouttes, et voici les chiffres obtenus :

\section{TABLEAU $\cdot$ XXXVI}

\begin{tabular}{|c|c|c|}
\hline \multicolumn{3}{|c|}{ Dilutions } \\
\hline \multicolumn{3}{|c|}{ Non diluée } \\
\hline Diluée & à & $1 / 2$ \\
\hline D & & $1 / 3$ \\
\hline$n$ & & $1 / 4$ \\
\hline 》) & & $1 / 5$ \\
\hline$n$ & & $1 / 6$ \\
\hline ” & & $1 / 10$ \\
\hline 》) & & $1 / 15$ \\
\hline " & & $1 / 20$ \\
\hline
\end{tabular}

Temps de prise

48-50 sec.

98- 100

145

200-205

260

310

465

690

920
Produit du temps de prise par le taux de la dilution
48-50
49-50
485
50-51
52
51
46,5
46
46

On peut dire que ces chiffres sont vraiment concordants surtout pour les premières dilutions, car au delà, le quotient diminue quelque peu et sur ce point nous nous trouvons d'accord avee GRTMMER et KRÜGER. Cela répond-il à un fait réel ou bien ne tient-il pas à la difficulté de saisir le moment exact de la coagulation, lorsque le temps de prise s'acçroît ? Nous pencherions peut-être vers cette dernière hypothèse. Quoi qu'il en soit, on voit que le produit: de la dilution de la présure par le temps de prise n'est pas loin d'être un nombre constant, du moins pour les dilutions qui ne sont pas exagérées.

ACTION DE LA TEMPÉRATURE SUR LA PRÉsURE ACIDIFIEE, NEUTRALISÉE ET ALCALINISEeE. - On a opéré avec de la présure diluée et de Ia présure non diluée, qu'on a chauffées pendant 15 minutes à $50^{\circ}, 55^{\circ}, 60^{\circ}, 65^{\circ}$, $70^{\circ}$ et $75^{\circ}$, sous réaction acide et après neutralisation vis-à-vis de la phtaléine du phénol, dans ce dernier cas done avec un $p H=8,2-8,3$.

L'alcalinisation à la phtaléine tuant absolument la présure avant $50^{\circ}$, il est indiqué de faire des essais à des températures moins élevées.

De la présure acide et de la présure neutralisée, ont été chauffées 15 minutes à $30^{\circ}, 35^{\circ}, 40^{\circ}, 45^{\circ}, 50^{\circ}, 55^{\circ}$ et $60^{\circ}$, puis refroidies après pour servir à l'emprésurage d'un lait dont $1^{\prime}$ acidité $\mathrm{D}$ était $18^{\circ}$ : 


\section{TABLEAU XXXVII.}

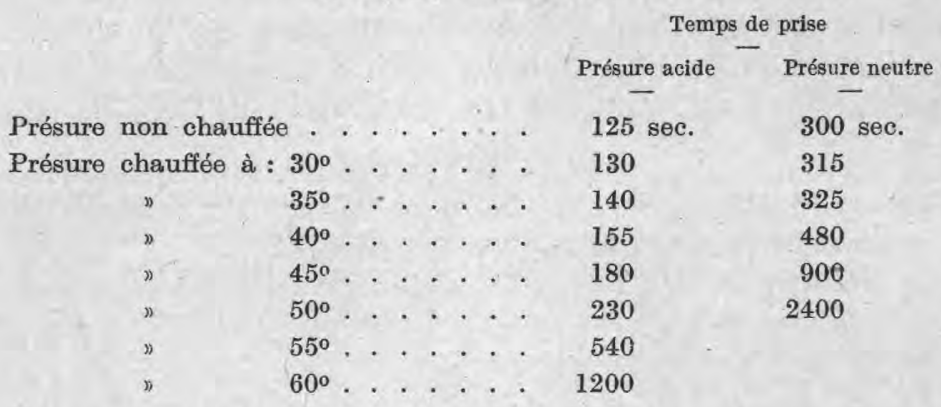

Avec la présure acide, le caillé ne commence à être mou que lorsque le chauffage dépasse $60^{\circ}$; avec la présure neutre, déjà par le chauffage à $45^{\circ}$. On voit par les résultats ci-dessus que l'altération de la présure commence à être sensible aux environs de $50^{\circ}$; à $55^{\circ}$, elle est nette, étant donné le retard très grand apporté à la coagulation du lait.

Si nous prenons une présure un peu plus forte, nous obtenons des résultats tout à fait semblables :

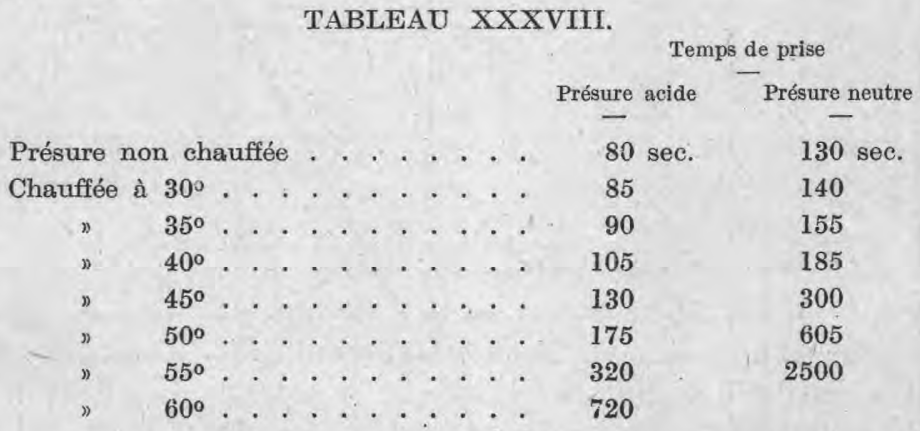

Comme c'est la règle, les caillés qui étaient fermes au début deviennent d'autant plus mous que la coagulation est plus lente.

LA NEUTRALISATION DE LA PRÉSURE ET LA REACIDIFICATION.Nous verrons plus loin l'influence grandement favorable de l'acidité du lait et des systèmes synthétiques sur la marche de l'emprésurage. Par contre, toute tendance à l'alcalımsation du lait nuira à l'action de la présure, la retardera, et ira même jusqu'à la supprimer. Mais, pour l'instant, ce n'est pas ce point que nous envisageons ; nous appellerons simplement l'attention sur l'influence de la neutralisation des solutions des présures elles-mêmes.

Une macération chlorhydrique à 4 pour 1000 de caillettes de veau est jetée sur un linge pour retenir les morceaux de cet estomac, puis filtrée sur papier, ce qui permet d'obtenir une liqueur jaunâtre très limpide. Cette solution est neutralisée après un essai préliminaire, très exactement par de la soude en présence de phtaléine du phénol. 
La présure ainsi neutre est réacidifiée, avec des quantités variables de présure contenant 1, 2, 4, 6 et 8 pour 1000 de $\mathrm{HCl}$. Cette réacidification entraîne, bien entendu, des dilutions dont il est tenu comptè sur toutes les liqueurs en expérience, afin que le taux de la présure originelle soit le même dans łoutes.

\section{GRAPHIQUE III.}

INFLUENCE DU TEMPS PENDANT LEQUEL LA PRÉSURE RESTE NEUTRALISÉE

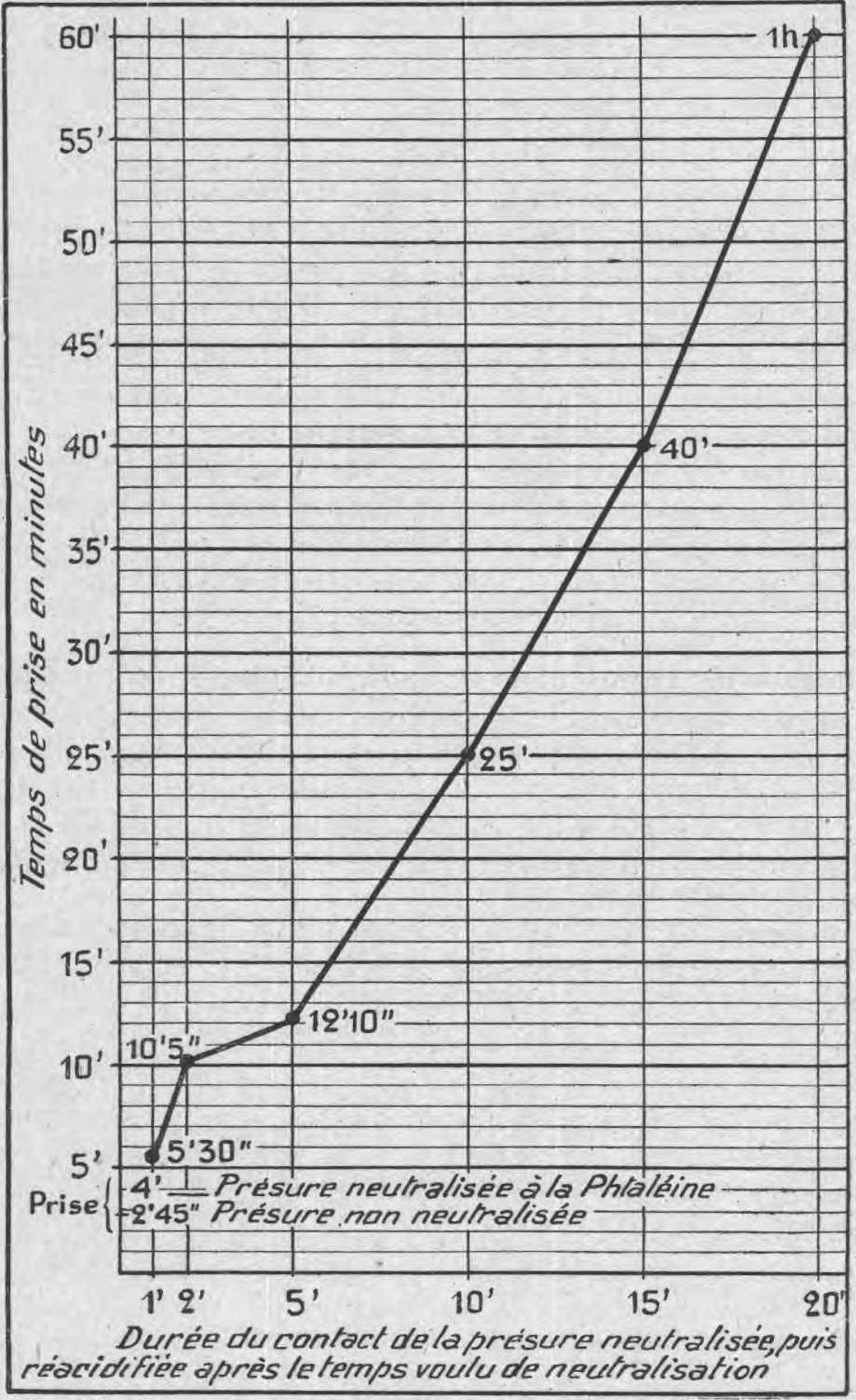


Un $1 / 2 \mathrm{~cm}^{3}$ de chaque dilution est additionné à $25 \mathrm{~cm}^{3}$ de lait et on porte au B-M à $40^{\circ}$.

Voici quels sont les temps de prise :

\section{TABLEAU XXXIX.}

\begin{tabular}{|c|c|c|c|c|c|c|c|c|c|}
\hline & résure & & & & & & & & Temps de prise \\
\hline Neutralis & sée. . . & & , & . . & . . & . & & . & . 275 sec. \\
\hline Acidifiée & : $1 / 1000$ & $\mathrm{HCl}$. & . & . & . . & . & . . & & 175 \\
\hline$"$ & $2 / 1009$ & $n$. & . & . & & . & & . & 145 \\
\hline ” & $4 / 1000$ & ” & . & . & . & . & . & . & 125 \\
\hline D & $6 / 1000$ & " & . & & & & & & 100 \\
\hline ” & $8 / 1000$ & " & . & . & . & & & & 90 \\
\hline
\end{tabular}

La présure originelle, avant toute neutralisation, et à la même dilution, faisait prendre la même quantité de lait en 2 minutes 20 secondes, soit 140 secondes. C'est approximativement la moitié du temps qu'emploie la préeure neutralisée, exactement à la phénol-phtaléne, pour coaguler la même quantité de lait.

Cette expérience suggestive nous permet de conclure que la simple neutralisation de la présure porte une atteinte sérieuse à son action sur le lait, elle a fait fléchir sa force de $50 \%$. La réacidification, même avec seulement 1 pour 1000 d'acide chlorhydrique, redonne à cette présure sa force initiale presque toute entière. En tout cas, nous saisissons parfaitement l'action de l'acidité sur laquelle nous aurons à maintes reprises, un peu plus loin, à appeler l'attention.

\section{Influence du temps dans la neutralisation et l'alcalinisation} de la présure. - Dans l'expérience qui précède, la réacidification de la solution de présure a suivi de peu sa neutralisation. Nous allons voir maintenant que si on laisse la présure pendant plusieurs minutes en liqueur neutre et a fortiori en liqueur légèrement alcaline, elle s'altère très vite, et la réacidification n'est plus à même de lui faire recouvrer sa force originelle.

A $10 \mathrm{~cm}^{8}$ de présure neutralisée, on ajoute $1 / 4 \mathrm{de}^{\mathrm{cm}} \mathrm{c}^{3}$ 'une solution de soude normale et on laisse en contact des temps variables : 1, 2, 5, 10, 15, 20 minutes, puis on traite ces présures par $1 / 2 \mathrm{~cm}^{3}$ d'acide chlorhydrique normal ; $1 / 4$ d'acide neutralisera done le $1 / 4$ de soude surajoutée, et finalement la solution de présure sera de nouveau acidulée. On réacidifiera la solution alcaline de présure juste avant de s'en servir pour en introduire la quantité voulue dans le lait.

Le graphique ci-contre montre l'altération profonde que fait subir la soude à la présure. 